\title{
Decreased concentration of adiponectin together with a selective reduction of its high molecular weight oligomers is involved in metabolic complications of myotonic dystrophy type 1
}

\author{
Aurora Daniele ${ }^{1,2,3}$, Anna De Rosa ${ }^{1}$, Mario De Cristofaro ${ }^{4}$, Maria Ludovica Monaco ${ }^{1,3}$, Mariorosario Masullo ${ }^{5}$, \\ Carola Porcile $^{6}$, Mario Capasso ${ }^{1}$, Gioacchino Tedeschi ${ }^{4}$, Giovannangelo Oriani ${ }^{1,6}$ and Alfonso Di Costanzo $^{6}$ \\ ${ }^{1}$ CEINGE Biotecnologie Avanzate Scarl, Via Gaetano Salvatore 486, 80145 Naples, Italy, ${ }^{2}$ Dipartimento di Scienze Ambientali, Seconda Università degli \\ Studi di Napoli, Caserta, Italy, ${ }^{3}$ IRCCS Fondazione SDN, Naples, Italy, ${ }^{4}$ Dipartimento di Scienze Neurologiche, Seconda Università degli Studi di Napoli, \\ Naples, Italy, ${ }^{5}$ Dipartimento di Studi delle Istituzioni e dei Sistemi Territoriali, Università degli Studi di Napoli 'Parthenope', Naples, Italy and \\ ${ }^{6}$ Dipartimento di Scienze per la Salute, Università degli Studi del Molise, Campobasso, Italy
}

(Correspondence should be addressed to A Daniele at CEINGE Biotecnologie Avanzate Scarl; Email: daniele@ceinge.unina.it)

\begin{abstract}
Objective: The hormone adiponectin exerts beneficial pleiotropic effects on biological and metabolic processes. Although a well-recognized insulin sensitizer, its characteristic has yet to be clearly defined. Myotonic dystrophy type 1 (DM1) is a rare genetic disorder that features muscle wasting and metabolic comorbidity, and patients have an increased risk of developing type 2 diabetes. We analyzed circulating levels of adiponectin and its oligomers to determine whether their expression correlates with metabolic alterations in DM1 patients.

Design and methods: We measured the anthropometric and biochemical features and three insulin resistance (IR) indices (homeostasis model assessment, quantitative insulin sensitivity check index, and McAuley) of 21 DM1 patients and of 82 age-, sex-, and weight-matched controls. In the blood samples of patients and controls, adiponectin levels were measured by ELISA, and its oligomers were characterized by using western blotting and gel filtration. The adiponectin gene was molecularly analyzed in patients.

Results: DM1 patients had significantly higher body mass index, waist circumference, triglycerides (TGs), glucose, tumor necrosis factor $\alpha$, and IR; conversely, they had significantly lower concentrations of total serum adiponectin with a selective, pronounced decrease of its high molecular weight (HMW) oligomers. There was a strong negative correlation between adiponectin and TGs in DM1 patients. Conclusions: Our results endorse the hypothesis that decreased expression of adiponectin together with a selective reduction of its HMW oligomers contributes to the worsening of IR and its metabolic complications in DM1 patients. These findings suggest that adiponectin and HMW oligomers may serve as biomarkers and are promising therapeutic agents for IR and its consequences in DM1.
\end{abstract}

European Journal of Endocrinology 165 969-975

\section{Introduction}

Adiponectin (ACRP30 or ADIPOQ) is a secretory protein exclusively synthesized by adipose tissue. Owing to its anti-inflammatory, antiatherogenic, and antidiabetic properties, ACRP30 exerts beneficial and protective effects on energy metabolism and cell differentiation and proliferation (1). Low concentrations of serum ACRP30 are related to two metabolic disorders, namely obesity and insulin resistance (IR) $(1,2)$. Moreover, low ACRP30 levels have been implicated in cancer pathogenesis (3). Single-nucleotide polymorphisms (SNPs) and adiponectin gene (ACDC or $A D I P O Q$ ) haplotypes have been associated with obesity, type 2 diabetes mellitus (T2DM), metabolic syndrome, and coronary artery disease (CAD) $(2,4,5)$.
The effects of ACRP30 are mediated by ADIPOR 1 and ADIPOR2 receptors, which are ubiquitous, although the former is mainly expressed in skeletal muscle and the latter reaches its highest levels in the liver (4). ACRP30 is a protein secreted in serum at high levels that vary depending on sex, body fat distribution, and metabolic status $(1,2,4)$. A striking feature of ACRP30 is the ability to assemble in various oligomeric complexes, namely trimers (low molecular weight (LMW)), hexamers (medium MW (MMW)), and oligomers larger than hexamers (high MW $(\mathrm{HMW}))(1,4,6)$. The state of ACRP30 oligomerization is critical for its signal transduction pathway and for the regulation of its biological activities $(1,4,6)$. A large body of evidence indicates that HMW oligomers are the main bioactive 
forms that mediate ACRP30's insulin-sensitizing effects and several metabolic abnormalities. The rare human adiponectin mutations G84R and G90S result in a decrease in HMW oligomer levels that are closely associated with $\mathrm{T} 2 \mathrm{DM}(4,6)$.

Myotonic dystrophy type 1 (DM1) is a multisystem autosomal dominant disorder characterized by progressive muscle wasting and weakness (7). The primary defect in DM1 is the expansion of a CTG triplet repeat in the $3^{\prime}$-UTR of the myotonic dystrophy protein kinase (DMPK) gene. The complex phenotype of DM1 is usually characterized by metabolic dysfunctions such as IR and hyperinsulinemia, hypertriglyceridemia, increased fat mass, and a fourfold higher risk of developing T2DM (8-11). The pathogenetic mechanism leading to these metabolic dysfunctions in DM1 remains unclear but it is closely linked to disorders of muscle cells containing reduced levels of insulin receptor and muscle-specific chloride-channel mRNAs, and elevated levels of tumor necrosis factor $\alpha(\mathrm{TNF} \alpha)(12-14)$.

To date, only two studies have evaluated ACRP30 expression in DM1 patients $(15,16)$, although DM1 is considered a good model for the study of metabolic perturbations in IR with adequate islet compensation. To determine whether the metabolic perturbations in DMI are associated with deranged adiponectin expression, we measured circulating levels of ACRP30 and characterized its oligomerization pattern. We also evaluated whether molecular alterations of the $A C D C$ gene were involved in the metabolic complications of the DM1 phenotype. Finally, we examined the correlations between total ACRP30 expression and several biochemical parameters in 21 DM1 patients and 82 age-, sex-, and weight-matched healthy controls.

\section{Subjects and methods}

\section{Subjects}

Twenty-one patients with the adult form of DM1 were recruited from outpatients attending the neurological clinic of the Second University of Naples (South Italy). Diagnoses were made based on clinical findings and genetic analyses according to a standard protocol (17). The severity of muscular involvement was assessed using the muscular impairment rating scale (17) and ranged from two (minimal signs) to five (severe weakness). Nine patients had normal electrocardiograms. The remaining 12 patients were affected by firstdegree atrioventricular block and/or bundle branch block. Conventional echocardiography revealed normal left ventricular dimensions, wall thickness, and global systolic function (left ventricular ejection fraction 50-77\%, mean \pm s.D., $64.1 \pm 8.7$ ). Left ventricular ejection fraction is a parameter for the diagnosis, prognosis, and treatment of various types of heart disease (18). No patient presented signs and/or symptoms of CAD. Eighty-two age-, sex-, and weightmatched healthy volunteers served as controls.

The anthropometric and biochemical features of the study participants are shown in Table 1. Height, weight, and waist circumference at umbilicus were measured while the subject was standing. Body mass index (BMI) was calculated as the body weight $(\mathrm{kg})$ divided by height (m) squared. Systolic and diastolic blood pressures were measured after the subject had been seated for at least 10 min. Creatinine clearance was estimated with the Cockroft-Gault formula, which is widely used in crosssectional studies, being easy to use, reproducible, and inexpensive (19).

Table 1 Anthropometric and biochemical characteristics of study participants. Data are expressed as mean (s.D.) and median.

\begin{tabular}{llll}
\hline Parameters & $\begin{array}{l}\text { Controls } \\
n=82\end{array}$ & $\begin{array}{l}\text { Patients } \\
n=21\end{array}$ & P values \\
\hline Age (years) & $39.2(15.8) ; 33.5$ & $44.5(16.9) ; 46.5$ & $\mathrm{NS}$ \\
Sex (male/female) & $43 / 39$ & $8 / 13$ & $\mathrm{NS}$ \\
Weight $(\mathrm{kg})$ & $68.2(13.4) ; 68.5$ & $69.9(10.7) ; 66.5$ & $\mathrm{NS}$ \\
BMI $\left(\mathrm{kg} / \mathrm{m}^{2}\right)$ & $23.2(2.9) ; 23.4$ & $25.7(3.6) ; 25.2$ & 0.001 \\
Waist circumference $(\mathrm{cm})$ & $84.9(11.6) ; 89$ & $97.4(8.6) ; 95$ & $<0.001$ \\
Total cholesterol $(\mathrm{mg} / \mathrm{dl})$ & $194.5(38.9) ; 190.0$ & $210.8(51.3) ; 198.5$ & $\mathrm{NS}$ \\
Triglycerides $(\mathrm{mg} / \mathrm{dl})$ & $95.2(53.9) ; 82.5$ & $244.1(186.0) ; 146$ & $<0.001$ \\
Glucose $(\mathrm{mg} / \mathrm{dl})$ & $81.6(11.7) ; 80.0$ & $108.5(42.2) ; 100.0$ & $<0.001$ \\
TNF $\alpha(\mathrm{pg} / \mathrm{ml})$ & $4.0(2.1) ; 3.6$ & $6.8(2.4) ; 6.8$ & $<0.001$ \\
Insulin t0 $(\mathrm{mU} / \mathrm{l})$ & $6.6(1.8) ; 6.0$ & $13.8(9.1) ; 10.8$ & $<0.001$ \\
HOMA & $1.3(0.2) ; 1.3$ & $3.6(2.2) ; 3.0$ & $<0.001$ \\
QUICKI & $0.4(0.0) ; 0.4$ & $0.33(0.0) ; 0.3$ & $<0.001$ \\
McAuley & $8.7(1.4) ; 9.2$ & $5.6(1.8) ; 5.5$ & $<0.001$ \\
Fibrinogen $(\mathrm{mg} / \mathrm{dl})$ & $274.7(44.6) ; 268.7$ & $336.7(73.7) ; 312.0$ & $<0.01$ \\
Estimated creatinine clearance $(\mathrm{ml} / \mathrm{min})$ & $103.1(71.7) ; 94.0$ & $97.4(29.1) ; 95.5$ & $\mathrm{NS}$ \\
Left ventricular ejection fraction $(\%)$ & $66.1(2.6) ; 65.9$ & $64.1(8.7) ; 65.0$ & $\mathrm{NS}$ \\
Total ACRP30 ( $\mu \mathrm{gg} / \mathrm{ml})$ & $29.0(7.9) ; 27.7$ & $22.0(9.5) ; 19.8$ & $<0.001$ \\
\hline
\end{tabular}

The statistical significance was established at $P<0.05$. NS, not significant; BMI, body mass index; HOMA, homeostasis model assessment; QUICKI, quantitative sensitivity check index. 
Informed consent was obtained from each patient and each volunteer. The study was approved by the Local Ethics Committee and was conducted in accordance with ethical principles stated in the most recent version of the Declaration of Helsinki.

\section{Laboratory measurements}

For laboratory studies, $2 \mathrm{ml}$ blood samples were collected after a $12 \mathrm{~h}$ overnight fast and immediately centrifuged at room temperature. Serum levels of total cholesterol, triglycerides (TGs), glucose, and fibrinogen were determined with standard enzymatic methods (Hitachi Modular, Roche). Serum TNF $\alpha$ and insulin were measured by immunoassays (Immunolight 2000, Medical System, Genova, Italy and Human TNF $\alpha$ Quantikine, R\&D Systems, Minneapolis, MN, USA respectively).

A standard oral glucose tolerance test (OGTT) was performed as described elsewhere (13). After a $12 \mathrm{~h}$ overnight fast, peripheral venous blood samples were collected before and 30,60, 120, and $180 \mathrm{~min}$ after ingestion of $75 \mathrm{~g}$ glucose. IR was estimated according to the homeostasis model assessment (HOMA), calculated as (fasting glucose $\times$ fasting insulin)/22.5 (20). The quantitative insulin sensitivity check index (QUICKI) and the McAuley index were calculated as described previously (20). In detail, QUICKI is calculated as $1 /(\log$ insulin $+\log$ glucose in $\mathrm{mg} / \mathrm{dl})$; McAuley calculated as exp [2.63-0.28 ln (insulin in mU/l)$0.31 \mathrm{ln}$ (TG in mmol/l)]. Patients were considered insulin resistant when HOMA was $\geq 2.6$, QUICKI $\leq 0.33$, and McAuley $\leq 5.8$.

\section{Measurement of serum ACRP30 by ELISA}

ACRP30 concentrations were measured with an ELISA using a polyclonal antibody produced in-house vs a human ACRP30 amino acid fragment $\left(\mathrm{H}_{2} \mathrm{~N}-\mathrm{ETTTQ}\right.$ GPGVLLPLPKG-COOH) as described previously (21). ACRP30 was measured three times in triplicate.

\section{Western blotting analysis}

Serum samples were purified using Aurum Affi-GEL Blue columns (Bio-Rad). Proteins were quantified with Bradford's method (Bio-Rad), and $10 \mu \mathrm{g}$ purified serum was treated in Laemmli buffer with and without $10 \mathrm{mmol} / \mathrm{l}$ dithiothreitol (DTT) and loaded on a $10 \%$ SDS-PAGE. The western blotting analysis was performed as described previously (21). All experiments were performed in triplicate.

\section{Gel filtration analysis}

The distribution of ACRP30 oligomers in serum was analyzed on a Superdex 200 10/300 GL column connected to a fast protein liquid chromatography system (Amersham Biosciences Biotech) as previously reported (21). Two hundred microliters of serum samples were fractionated at $0.5 \mathrm{ml} / \mathrm{min}$ using a PBS containing $5 \mathrm{mM}$ DTT as elution buffer. Fractions $(250 \mu \mathrm{l})$ were collected and the concentration of ACRP30 oligomers was measured by ELISA as reported above; the oligomeric composition was assessed by western blotting. The column was calibrated using apo-ferritin (440 kDa), amylase (200 kDa), and glyceraldehyde-3-P dehydrogenase (160 and $46 \mathrm{kDa})$ as molecular weight standards (Sigma-Aldrich).

\section{Molecular analysis of the ACDC gene}

Blood samples $(2 \mathrm{ml})$ were collected by venipuncture in EDTA and genomic DNA was extracted using a standard salting out/ethanol precipitation. Exon regions, the exon-intron boundaries, and the promoter region of $A C D C$ gene were amplified with an in-house primer set using the PCR protocol, as described previously (5). PCR products were electrophoresed on a $1 \%$ agarose gel and both strands were sequenced with an automated procedure using the 3100 Genetic analyzer (Applied Biosystems, Foster City, CA, USA). PCR fragments were sequenced with the same primers used for PCR amplification.

\section{Statistical analysis}

Data were analyzed using the SPSS (v 10.0) Software package (SPSS, Inc., Chicago, IL, USA). Variables were examined for outliers and extreme values by the box and normal quantile-quantile plots, for normal distribution by the Kolmogorov-Smirnov and Shapiro-Wilk tests, and for homogeneity of variance by the Levene test. When normal distribution could not be accepted, variable transformations (square, square root, logarithmic, reciprocal of square root, or reciprocal transformations) were reviewed. Since normality was not reached for most variables, we used the following nonparametric tests: the Mann-Whitney $U$ test for group differences and the Spearman rank coefficient $\left(r_{\mathrm{s}}\right)$ for correlation analysis. The $\chi^{2}$ test was used to compare sex ratio. To overcome confounder effects of variables on adiponectin levels, we used a general linear model for analysis of covariance (ANCOVA) and stepwise multiple regression analysis ( $F$ to enter $>3.84)$. Statistical significance was established at $P<0.05$.

\section{Results}

The anthropometric and biochemical features of DM1 patients and healthy controls are listed in Table 1. DM1 patients and the age-, sex-, and weight-matched controls had comparable systolic/diastolic pressure (data not shown), total cholesterol, estimated creatinine 
clearance, and left ventricular ejection fraction, whereas DM1 patients had significantly higher BMI, waist circumference, and TG, glucose, TNF $\alpha$, insulin, and fibrinogen levels $(P<0.001)$. HOMA, QUICKI, and McAuley indicated that DM1 patients had significantly higher IR than controls $(P<0.001)$. OGTT, performed in 13 DM1 patients, revealed serum levels of glucose and insulin indicative of glucose intolerance and hyperinsulinemia (data not shown). In addition, DM1 patients had significantly lower concentrations of total ACRP30 $(P<0.001)$. The difference in total ACRP30, TG, and TNF $\alpha$ levels between controls and patients persisted after adjustment for age, sex, and BMI (data not shown).

ANCOVA was performed to remove confounder effects of variables that influence ACRP30 levels. The analysis, adjusted for age, sex, BMI, waist circumference, total cholesterol, TG, glucose, insulin, HOMA, McAuley, estimated creatinine clearance, and left ventricular ejection fraction, confirmed the significant difference in ACRP30 concentrations between the two groups $(F=4.2 ; \mathrm{df} 1.66 ; P=0.045)$. Total ACRP30 levels correlated negatively with age $\left(r_{\mathrm{s}}=-0.46\right.$, $P<0.001)$, BMI $\left(r_{\mathrm{s}}=-0.37, P=0.001\right)$, and waist circumference $\left(r_{\mathrm{s}}=-0.40, P<0.001\right)$ in the control group. Total ACRP30 levels correlated negatively with TG levels $\left(r_{\mathrm{s}}=-0.59, P=0.006\right)$ in the DM1 group. Multiple regression analysis with age, sex, BMI, waist circumference, total cholesterol, TG, glucose, insulin, HOMA, McAuley, estimated creatinine clearance, and left ventricular ejection fraction as independent variables showed that total ACRP30 levels remained negatively correlated with age $\left(r^{2}=0.18, F=8.3\right.$, $P=0.007)$ in the control group and with TG $\left(r^{2}=\right.$ $0.22, F=5.14, P=0.036)$ in the DM1 group.

To verify the decrease in ACRP30 serum levels reported above, we examined the serum of control subjects and DM1 patients by western blotting in nonreducing and reducing conditions. As shown in Fig. 1A, a single band of molecular weight $>250 \mathrm{kDa}$ corresponding to ACRP30 appeared under non-reducing conditions. Densitometric analysis (Fig. 1B) showed that the total amount of ACRP30 was 1.7-fold lower in DM1 patients than in controls $(21721 \pm 11120$ pixels vs $36123 \pm 8674$ pixels respectively; $P<0.01)$. These results are similar to the results of ELISA and confirm that the total amount of ACRP30 was significantly lower in the serum of DM1 patients than in control subjects. Western blotting, performed under reducing conditions, revealed three major molecular mass species corresponding to LMW, MMW, and HMW ACRP30 oligomers $(\sim 60, \sim 150$, and $>250 \mathrm{kDa}$ respectively). Figure 1C shows the distribution of ACRP30 oligomers in the serum of representative controls (lanes 1 and 2) and DM1 patients (lanes 3-8). The pattern of ACRP30 oligomers differed between most DM1 patients and controls, the level of HMW and MMW oligomers being very low in patients. Western blotting analysis revealed
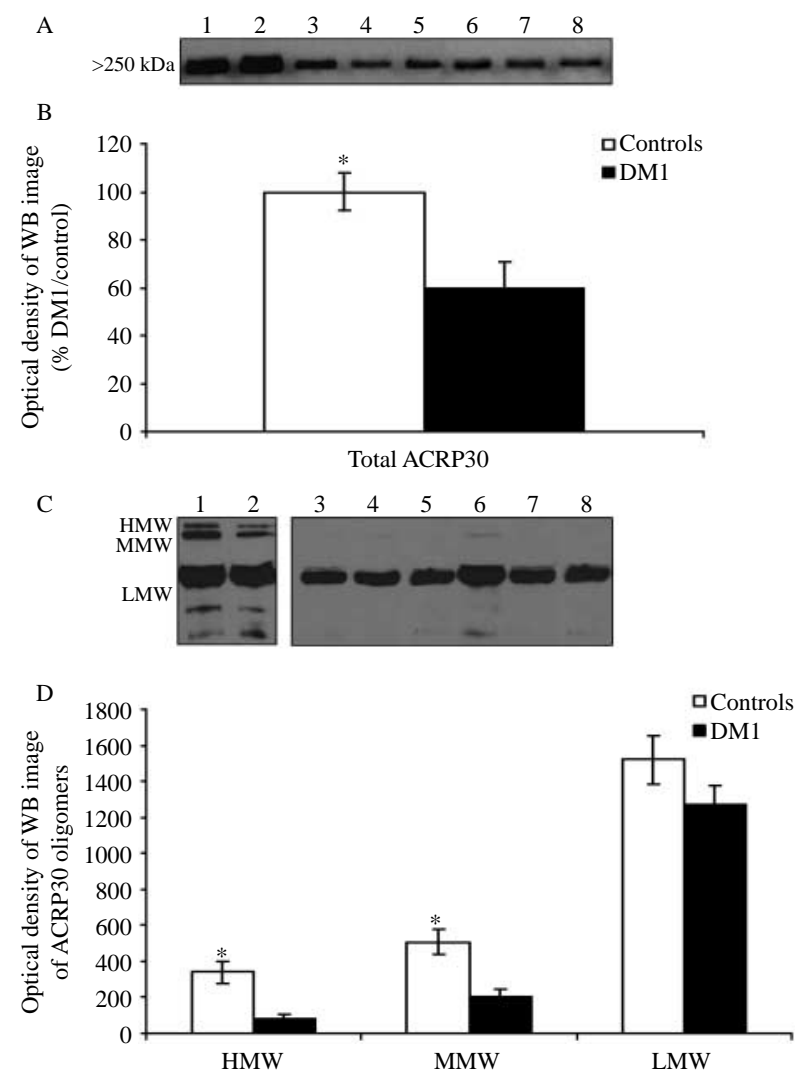

Figure 1 Western blotting analysis of serum ACRP30 in controls and DM1 patients in non-reducing ( $A$ and $B$ ) and reducing ( $C$ and $D)$ conditions. (A) Representative blot image for total serum ACRP30 in controls (lanes 1 and 2) and in DM1 patients (lanes 3-8).

(B) Graphic representation of pixel analysis performed with ImageJ Software showing that total serum ACRP30 expression is significantly lower in DM1 patients than in controls $\left({ }^{\star} P<0.01\right)$. Values are reported as pixel mean \pm SD of three experiments expressed as percentage vs control. (C) Representative blot showing the ACRP30 oligomers (HMW, MMW, and LMW) in the serum of controls (lanes 1 and 2) and DM1 patients (lanes 3-8). (D) Graphic representation of pixel analysis performed using ImageJ Software (available from http://rsbweb.nih.gov.ij/) showing that HMW and MMW expression is significantly lower in DM1 patients than in controls $\left({ }^{\star} P<0.01\right)$. Values are reported as mean $\pm S D$ of three experiments.

that the difference in total ACRP30 levels between the two groups in non-reducing conditions is due to a lower level of HMW and MMW oligomers in DM1 patients vs controls. In particular, HMW oligomers were more than fourfold higher in controls than in DM1 patients, and MMW oligomers were 2.5-fold higher in controls than in DM1 patients. LMW oligomers did not differ between the two groups (Fig. 1D). The total amount of ACRP30 (i.e. the sum of the HMW, MMW, and LMW oligomers) was 1.6-fold lower in DM1 than in controls. Taken together, our results indicate that the lower amount of ACRP30 in most DM1 patients was primarily due to a decrease in HMW oligomers and secondarily to a decrease in MMW, whereas LMW ACRP30 oligomers remained unchanged. 
To study the association of ACRP30 with DM1 in more detail, we evaluated the distribution of its oligomers in the serum of DM1 patients and controls by FPLC-gel filtration chromatography. Figure 2A shows the oligomerization state of the fractions of two representative DM1 patients and a representative control obtained by ELISA. The western blotting analysis of these fractions confirmed that ACRP30 HMW oligomers were more abundant in controls than in DM1 patients (Fig. 2B).

The molecular analysis of SNPs in the ACDC gene of DM1 patients revealed six variants previously reported in Caucasian populations (5). All variants were detected in the heterozygous state and four of them were located in the promoter region (c. $-11426 \mathrm{~A}>\mathrm{G}$, c. $-11391 \mathrm{G}>\mathrm{A}$, c. $-11377 \mathrm{C}>\mathrm{G}$, and c. -11156 insCA), one in exon 2 (c.45 T>G p.G15G), and one in intron 2 (c.214+62G $>$ T; data not shown).

\section{Discussion}

To our knowledge, this is the first study to characterize the distribution of circulating ACRP30 oligomers in patients affected by DM1. We demonstrate that ACRP 30 expression is altered in these patients. In particular, total serum ACRP30 was significantly lower in DM1 patients than in controls due to a pronounced, selective decrease in its HMW oligomers. BMI, waist circumference, TG, insulin, TNF $\alpha$, and fibrinogen were significantly higher in our DM1 patients than in age-, sex-, and body weight-matched controls. Their glucose levels were within normal range, although the results of OGTT were indicative of IR and glucose intolerance. Moreover, in patients we found a strong negative correlation between ACRP30 and TG levels, but no

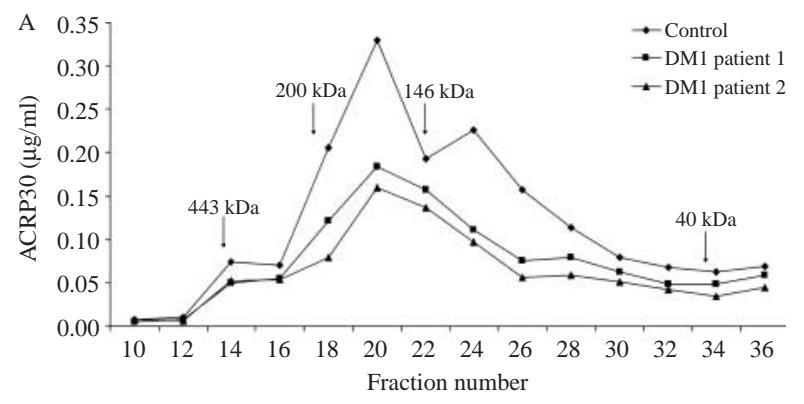

B

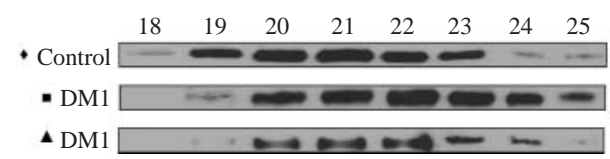

Figure 2 Analysis of oligomerization state of ACRP30 by FPLC-gel filtration. An aliquot of each collected fraction was subjected to ELISA and western blotting analysis. (A) Graphic representation of the ELISA of one control and two DM1 patients and (B) the relative western blotting images of FPLC fractions showing that the expression of HMW and of MMW, albeit to a lesser extent, is lower in DM1 patients, whereas there was no between-group difference in the expression of LMW oligomers. significant association with the other parameters was analyzed. In our control group, there was a significant negative correlation between ACRP30 and age, waist circumference, and BMI, but only age remained significantly correlated after multiple regression analysis.

The clinical features of DM1 patients are often complicated by metabolic abnormalities and hormonal deregulation including IR $(8-11,22)$. ACRP30 and HMW oligomers are potent insulin sensitizers. In fact, the increase in serum ACRP30 by genetic and/or pharmacological approaches can alleviate metabolic dysfunctions $(23,24)$.

Here, we show that ACRP30 and its HMW oligomers are involved in the metabolic phenotype of DM1 patients. Our results are consistent with the report of Matsumura et al. (15) who showed that that ACRP30 concentrations were lower in patients with glucose concentrations comparable to those of our patients (i.e. between 90 and $110 \mathrm{mg} / \mathrm{dl}$ ). They are also in agreement with Abe et al. (16) who reported that serum ACRP30 concentrations improved in two DM1 patients with T2DM after treatment with pioglitazone. However, these two earlier studies did not measure the expression of HMW oligomers, which is the oligomer that accounts for most of the biological effects of ACRP30 $(4,6)$. In this study, we found a pronounced and preferential reduction of HMW oligomers in the serum of DM1 patients. Hypoadiponectinemia and the inability to form HMW oligomers can be due to SNPs and/or mutations of the ACDC gene $(2,4)$. In particular, two rare mutations, G84R and G90S, are closely associated with IR and T2DM $(4,6,25)$. In our molecular analysis of the ACDC gene of DM1 patients (data not shown), we found only previously described SNPs $(2,5)$ and we are unable to determine whether they contribute to the genetic background of the patients' metabolic complication due to our small sample size. However, a number of studies have shown that CAD, weight gain, and IR are characterized by low concentrations of HMW and that these oligomers are more closely correlated with HOMA alterations and metabolic syndrome than total ACRP30 (25-27). It has recently been reported that a decrease in HMW oligomers is an independent risk factor for progression to T2DM in Japanese Americans and a predictor of CAD $(25,27,28)$. Thus, the decrease in total ACRP30 we observed, associated with the preferential reduction of HMW oligomers, may help to explain the decreased insulin sensitivity in patients affected by DM1.

It is not known why ACRP30 decreases in DM1 patients, but it is well established that elevated levels of insulin and TG are associated with low total ACRP30 levels $(25,29)$. Our DM1 patients had a large waist circumference and elevated TG levels (which correlated negatively with ACRP30 levels) as reported for subjects with primary hypertriglyceridemia $(30,31)$. 
Our DM1 patients had high levels of TNF $\alpha$; a potent inflammatory cytokine affects the function of not only muscle but also adipose tissues and thus induces IR and obesity (13, 14). In particular, TNF $\alpha$ inhibits the expression of ACRP30 and vice versa (32-34). Therefore, in DM1 patients, one could envisage a vicious cycle of IR, altered muscle and adipose tissue functions, and hypoadiponectinemia in which each of these factors strengthen the others. It is conceivable that this vicious cycle could be interrupted by exogenously increasing ACRP30 levels, which would result in a reduction of the metabolic complications of DM1.

A limitation of our study is the sample size, because DM1 is a rare disease. Nevertheless, ACRP30 levels in our patients were significantly lower than in controls even after adjusting for several confounding variables.

In conclusion, our results demonstrate that DM1 patients are characterized by low levels of total serum ACRP30 and by a pronounced and selective decrease in HMW oligomers. This preferential decrease in HMW oligomers is of interest because HMW oligomers are better correlated with insulin sensitivity than total ACRP30 - a finding suggesting that their reduction could contribute to IR in DM1, as reported for other diseases with metabolic alterations similar to those found in DM1 (25-27). However, although it remains to be established whether ACRP30 is directly involved in IR in DM1 patients, measurement of its serum levels might represent an additional criterion for the staging of metabolic abnormalities in DM1. Further studies are needed to better understand the role of ACRP30 in DM1, but our findings indicate that ACRP30, and in particular HMW oligomers, may be used as biomarkers of metabolic perturbations in DM1 patients and represent promising therapeutic agents against IR and its complications.

\section{Declaration of interest}

The authors declare that there is no conflict of interest that could be perceived as prejudicing the impartiality of the research reported.

\section{Funding}

This study was supported by the Regione Campania (DGRC n. 1901 del 22/12/2009), the MIUR (grant number: PS35-126/IND), the IRCCS-SDN Foundation, and the Ministero della Salute.

\section{Acknowledgements}

We thank Rita Ciaramella for her invaluable collaboration and Jean Ann Gilder (Scientific Communication srl, Naples, Italy) for revising and editing the text.

\section{References}

1 Brochu-Gaudreau K, Rehfeldt C, Blouin R, Bordignon V, Murphy BD \& Palin MF. Adiponectin action from head to toe. Endocrine 201037 11-32. (doi:10.1007/s12020-009-9278-8)
2 Gable DR, Hurel SJ \& Humphries SE. Adiponectin and its gene variants as risk factors for insulin resistance, the metabolic syndrome and cardiovascular disease. Atherosclerosis $2006 \mathbf{1 8 8}$ 231-244. (doi:10.1016/j.atherosclerosis.2006.02.010)

3 Lang K \& Ratke J. Leptin and adiponectin: new players in the field of tumor cell and leukocyte migration. Cell Communication and Signaling 2009727 . (doi:10.1186/1478-811X-7-27)

4 Kadowaki T, Yamauchi T, Kubota N, Hara K, Ueki K \& Tobe K. Adiponectin and adiponectin receptors in insulin resistance, diabetes, and the metabolic syndrome. Journal of Clinical Investigation 2006116 1784-1792. (doi:10.1172/JCI29126)

5 Daniele A, Cammarata R, Pasanisi F, Finelli C, Salvatori G, Calcagno G, Bracale R, Labruna G, Nardelli C, Buono P, Sacchetti L, Contaldo F \& Oriani G. Molecular analysis of the adiponectin gene in severely obese patients from Southern Italy. Annals of Nutrition and Metabolism 2008 53 155-161. (doi:10.1159/000172976)

6 Wang Y, Lam KS, Yau MH \& Xu A. Post-translational modifications of adiponectin: mechanisms and functional implications. Biochemical Journal 2008409 623-633. (doi:10.1042/ BJ20071492)

7 Machuca-Tzili L, Brook D \& Hilton-Jones D. Clinical and molecular aspects of the myotonic dystrophies: a review. Muscle and Nerve 200532 1-18. (doi:10.1002/mus.20301)

8 Llagostera E, Catalucci D, Marti L, Liesa M, Camps M, Ciaraldi TP, Kondo R, Reddy S, Dillmann WH, Palacin M, Zorzano A, RuizLozano P, Gomis R \& Kaliman P. Role of myotonic dystrophy protein kinase (DMPK) in glucose homeostasis and muscle insulin action. PLoS ONE 20072 e1134. (doi:10.1371/journal.pone. 0001134)

9 Savkur RS, Philips AV \& Cooper TA. Aberrant regulation of insulin receptor alternative splicing is associated with insulin resistance in myotonic dystrophy. Nature Genetics 200129 40-47. (doi:10. 1038/ng704)

10 Perseghin G, Comola M, Scifo P, Benedini S, De Cobelli F, Lanzi R, Costantino F, Lattuada G, Battezzati A, Del Maschio A \& Luzi L. Postabsorptive and insulin-stimulated energy and protein metabolism in patients with myotonic dystrophy type 1. American Journal of Clinical Nutrition $2004 \mathbf{8 0} 357-364$.

11 Johansson A, Carlström K, Ahrén B, Cederquist K, Krylborg E, Forsberg H \& Olsson T. Abnormal cytokine and adrenocortical hormone regulation in myotonic dystrophy. Journal of Clinical Endocrinology and Metabolism 2000 85 3169-3176. (doi:10. 1210/jc.85.9.3169)

12 Day JW \& Ranum LPW. RNA pathogenesis of the myotonic dystrophies. Neuromuscular Disorders 200515 5-16. (doi:10. 1016/j.nmd.2004.09.012)

13 Fernández-Real JM, Molina A, Broch M, Ricart W, Gutiérrez C, Casamitjana R, Vendrell J, Soler J \& Gómez-Sáez JM. Tumor necrosis factor system activity is associated with insulin resistance and dyslipidemia in myotonic dystrophy. Diabetes $1999 \mathbf{4 8}$ 1108-1112. (doi:10.2337/diabetes.48.5.1108)

14 Zhang L, Lee JE, Wilusz J \& Wilusz CJ. The RNA-binding protein CUGBP1 regulates stability of tumor necrosis factor mRNA in muscle cells: implications for myotonic dystrophy. Journal of Biological Chemistry 2008283 22457-22463. (doi:10.1074/ jbc.M802803200)

15 Matsumura T, Iwahashi H, Funahashi T, Takahashi MP, Saito T, Yasui K, Saito T, Iyama A, Toyooka K, Fujimura H \& Shinno S. A cross-sectional study for glucose intolerance of myotonic dystrophy. Journal of the Neurological Sciences 2009276 60-65. (doi:10. 1016/j.jns.2008.08.037)

16 Abe H, Mita T, Kudo K, Funayama T, Tokoro M, Kaga H, Ikeda F, Kanazawa A, Hirose T, Kawamori R \& Wada H. Dramatic improvement of blood glucose control after pioglitazone treatment in poorly controlled over-weight diabetic patients with myotonic dystrophy. Endocrine Journal 200956 911-913. (doi:10.1507/ endocrj.K09E-122)

17 Di Costanzo A, Santoro L, de Cristofaro M, Manganelli F, Di Salle F \& Tedeschi G. Familial aggregation of white matter lesions in myotonic dystrophy type 1. Neuromuscular Disorders 200818 299-305. (doi:10.1016/j.nmd.2008.01.008) 
18 Shaw LJ, Peterson ED, Kesler K, Hasselblad V \& Califf RM. A metaanalysis of predischarge risk stratification after acute myocardial infarction with stress electrocardiographic, myocardial perfusion, and ventricular function imaging. American Journal of Cardiology 199678 1327-1337. (doi:10.1016/S0002-9149(96) 00653-4)

19 Wijeysundera DN, Rao V, Beattie WS, Ivanov J \& Karkouti K. Evaluating surrogate measures of renal dysfunction after cardiac surgery. Anesthesia and Analgesia 200396 1265-1273. (doi:10. 1213/01.ANE.0000056824.69668.33)

20 Ascaso JF, Pardo S, Real JT, Lorente RI, Priego A \& Carmena R. Diagnosing insulin resistance by simple quantitative methods in subjects with normal glucose metabolism. Diabetes Care 200326 3320-3325. (doi:10.2337/diacare.26.12.3320)

21 Daniele A, Cammarata R, Masullo M, Nerone G, Finamore F, D'Andrea M, Pilla F \& Oriani G. Analysis of adiponectin gene and comparison of its expression in two different pig breeds. Obesity 200816 1869-1874. (doi:10.1038/oby.2008.275)

22 Rakocevic Stojanovic V, Peric S, Lavrnic D, Popovic S, Ille T, Stevic Z, Basta I \& Apostolski S. Leptin and the metabolic syndrome in patients with myotonic dystrophy type 1. Acta Neurologica Scandinavica 2010121 94-98. (doi:10.1111/j.16000404.2009.01237.x)

23 Sulpice T, Prunet-Marcassus B, Molveaux C, Cani PD, Vitte PA, Graber P, Dreano M \& Burcelin R. An adiponectin-like molecule with antidiabetic properties. Endocrinology $2009 \quad 1504493-$ 4501. (doi:10.1210/en.2008-1768)

24 Hirose H, Yamamoto Y, Seino-Yoshihara Y, Kawabe H \& Saito I. Serum high-molecular-weight adiponectin as a marker for the evaluation and care of subjects with metabolic syndrome and related disorders. Journal of Atherosclerosis and Thrombosis 201017 1201-1211. (doi:10.5551/jat.6106)

25 Nishida M, Funahashi T \& Shimomura I. Pathophysiological significance of adiponectin. Medical Molecular Morphology 2007 40 55-67. (doi:10.1007/s00795-007-0366-7)

26 Nakashima R, Kamei N, Yamane K, Nakanishi S, Nakashima A \& Kohno N. Decreased total and high molecular weight adiponectin are independent risk factors for the development of type 2 diabetes in Japanese-Americans. Journal of Clinical Endocrinology and Metabolism 200691 3873-3877. (doi:10.1210/jc.20061158)

27 Inoue T, Kotooka N, Morooka T, Komoda H, Uchida T, Aso Y, Inukai T, Okuno T \& Node K. High molecular weight adiponectin as a predictor of long-term clinical outcome in patients with coronary artery disease. American Journal of Cardiology $2007 \mathbf{1 0 0}$ 569-574. (doi:10.1016/j.amjcard.2007.03.062)

28 Aso Y, Yamamoto R, Wakabayashi S, Uchida T, Takayanagi K, Takebayashi K, Okuno T, Inoue T, Node K, Tobe T, Inukai T \& Nakano Y. Comparison of serum high-molecular weight (HMW) adiponectin with total adiponectin concentrations in type 2 diabetic patients with coronary artery disease using a novel enzyme-linked immunosorbent assay to detect HMW adiponectin. Diabetes 200655 1954-1960. (doi:10.2337/db05-1525)

29 Matsubara M, Maruoka S \& Katayose S. Decreased plasma adiponectin concentrations in women with dyslipidemia. Journal of Clinical Endocrinology and Metabolism 2002872764 2769. (doi:10.1210/jc.87.6.2764)

30 Koh KK, Quon MJ, Lim S, Lee Y, Sakuma I, Lee YH, Han SH \& Shin EK. Effects of fenofibrate therapy on circulating adipocytokines in patients with primary hypertriglyceridemia. Atherosclerosis 2011 214 144-147. (doi:10.1016/j.atherosclerosis.2010.10.023)

31 Gómez Rosso L, Meroño T, Benítez MB, Lee Y, Sakuma I, Lee YH, Han SH \& Shin EK. Low adiponectin levels in primary hypertriglyceridemic male patients. Nutrition, Metabolism, and Cardiovascular Diseases 200919 135-139. (doi:10.1016/j. numecd.2008.02.008)

32 Cawthorn WP \& Sethi JK. TNF-alpha and adipocyte biology. FEBS Letters 2008582 117-131. (doi:10.1016/j.febslet.2007.11.051)

33 Hajri T, Tao H, Wattacheril J, Marks-Shulman P \& Abumrad NN. Regulation of adiponectin production by insulin: interactions with tumor necrosis factor- $\alpha$ and interleukin-6. American Journal of Physiology. Endocrinology and Metabolism 2011300 350-360. (doi:10.1152/ajpendo.00307.2010)

34 Degawa-Yamauchi M, Moss KA, Bovenkerk JE, Shankar SS, Morrison CL, Lelliott CJ, Vidal-Puig A, Jones R \& Considine RV. Regulation of adiponectin expression in human adipocytes: effects of adiposity, glucocorticoids, and tumor necrosis factor alpha. Obesity Research 200513 662-669. (doi:10.1038/oby.2005.74)

Received 16 June 2011

Revised version received 17 September 2011

Accepted 28 September 2011 